\title{
MÉTRICAS DE DESEMPENHO DE ESCOLAS DE ENSINO FUNDAMENTAL CICLADAS E NÃO CICLADAS
}

IVANETE BELLUCCI PIRES DE ALMEIDA FRANCISCO CARLOS BENEDETTI

\section{RESUMO}

A pesquisa que originou o presente texto mediu a eficiência das escolas públicas cicladas e não cicladas de ensino fundamental centrando-se nos municípios de Campinas, Belo Horizonte e Rio de Janeiro. Variáveis foram construídas para entender a influência do trabalho pedagógico do professor em relação ao tipo de escola (ciclada e não ciclada) e, simultaneamente, interessou-nos verificar a associação desse trabalho docente com outras variáveis: (a) as variáveis de proficiência média em leitura e matemática dos alunos, que foram medidas em dois momentos distintos de suas vidas escolares, a saber, na $1^{\underline{a}}$ série e na $3^{\underline{a}}$ série; e (b) a variável nível socioeconômico, investigada junto a um questionário respondido pelos pais. As associações entre tais variáveis nos permitiram a obtenção de níveis de eficiência das escolas.

PALAVRAS-CHAVE ESTUDO LONGITUDINAL • EFICIÊNCIA

- ANÁLISE DE DADOS • ENSINO FUNDAMENTAL • DESEMPENHO ESCOLAR. 


\section{RESUMEN}

La investigación que originó el presente texto midió la eficacia de las escuelas públicas "cicladas" y "no cicladas" de enseñanza fundamental, centrándose en los municipios de Campinas, Belo Horizonte y Rio de Janeiro. Se construyeron variables para entender la influencia del trabajo pedagógico del profesor en relación con el tipo de escuela ("ciclada" y "no ciclada") y, al mismo tiempo, nos interesó verificar la asociación de ese trabajo docente con otras variables: a) las variables de rendimiento medio de los alumnos en lectura y matemática, que fueron medidas en primero y tercer grado, dos momentos distintos de sus vidas escolares; b) la variable del nivel socioeconómico, investigada por medio de un cuestionario respondido por los padres. Las asociaciones entre esas variables nos permitieron obtener niveles de eficacia de las escuelas.

PALABRAS CLAVE ESTUDIO LONGITUDINAL • EFICIENCIA

- ANÁLISIS DE DATOS • ENSEÑANZA FUNDAMENTAL

-DESEMPEÑO ESCOLAR.

\section{ABSTRACT}

The study that gave rise to this article measured the effectiveness of cycled and non-cycled public elementary schools located in Campinas, Belo Horizonte and Rio de Janeiro. We are not concerned with specific concepts involved in the evaluation process of reading and mathematics, but we focus on the quantitative performance in these disciplines by many students of the abovementioned cities, and analyzed them by schools. Variables were built to help understand the influence of the teacher's pedagogical practice in relation to the type of school (cycled and non-cycled). Simultaneously, we were interested in establishing the association between this pedagogical practice and other variables, for instance: (a) average proficiency variables of students in reading and in mathematics, with data collected at two different moments in their educational lives, the 1st and 3rd grades; (b) the socioeconomic level variable, the data of which were collected in a survey with the parents. The associations between these variables helped us in the evaluation of the schools' efficiency. 


\section{INTRODUÇÃO}

O presente texto tem por objetivo discutir as potencialidades de pesquisas quantitativas em Educação, mais especificamente, no ensino fundamental público, e apresentar um modelo de avaliação com perfil estatístico, possível de ser articulado com uma abordagem qualitativa. A discussão baseia-se nos resultados obtidos na pesquisa de doutorado de Almeida (2009), cuja hipótese é que escolas públicas cicladas têm, em relação às não cicladas, desempenhos no mínimo semelhantes nos processos gerais de ensino e aprendizagem em Leitura e Matemática, ao menos nos polos de Belo Horizonte, Campinas e Rio de Janeiro, nos quais se realizou a pesquisa.

\section{CONTEXTO E METODOLOGIA DA PESQUISA}

O caminho metodológico percorrido seguiu os seguintes passos:

\section{AS ESCOLAS ESTUDADAS. O BANCO GERES}

Os dados dos participantes deste estudo foram extraídos do Projeto

Geres: Geração Escolar 2005, projeto concebido, primordialmente, 
para estudar o valor agregado das escolas ao longo dos primeiros anos de escolarização no Brasil.

Em nossa pesquisa, no período de 2005 a 2006, trabalhamos com 12.678 alunos nos polos de Campinas (SP), Belo Horizonte (MG) e Rio de Janeiro (RJ). As quantidades de alunos avaliados em leitura e matemática foram distribuídas por polo, rede e tipo de escola (ciclada e não ciclada), sendo 10.201 pertencentes ao grupo de escolas cicladas e 2.477 , ao de não cicladas.

Essa diferença numérica parece ser indesejável, porém a pesquisa se concentrou nos rendimentos por escola, contemplando 110 escolas públicas organizadas em ciclos e séries. O motivo para a escolha desses três polos, diante de outros possíveis, é que eles possuíam uma maior quantidade de escolas cicladas.

\section{APRESENTAÇÃO DAS VARIÁVEIS ESTUDADAS}

Estabelecido o contexto e os participantes da pesquisa, partimos para a seleção das variáveis relevantes para o objetivo visado, qual seja, analisar a eficiência das escolas cicladas e não cicladas dos três polos pesquisados.

Procuramos trabalhar com variáveis que a própria literatura em Educação aponta como possíveis condicionantes de eficiência. Foram elas: (a) variável nível socioeconômico (NSE), calculado por meio do critério Abep/Abipeme; (b) variável trabalho pedagógico do professor; (c) variável proficiência média das escolas em leitura e matemática (resultado das proficiências dos alunos ao responder aos dois testes analisados).

\section{ANÁLISE POR ENVOLTÓRIA DE DADOS}

A Análise por Envoltória de Dados (DEA) foi usada para aferir a eficiência das escolas analisadas, ou seja, forneceu as informações finais do estudo. Essa ferramenta operacional recebeu os dados "refinados", após terem sido trabalhados e estudados de acordo com as variáveis descritas no item anterior.

\section{DETALHAMENTO DAS VARIÁVEIS}

Antes de tratarmos da forma como foram obtidos os dados, é necessário apontar, ainda que sucintamente, as características gerais dos instrumentos de coleta de dados. Eles foram utilizados de forma praticamente simultânea: durante a coleta de dados junto aos estudantes em novembro de 2005, ou seja, em 
meio às realizações das avaliações em Leitura e Matemática, um questionário foi aplicado aos pais, a fim de obter informações para a composição da variável NSE; e os professores responderam a questões sobre suas práticas pedagógicas, de modo que seus desempenhos pudessem ser mensuráveis.

Assim, a partir de cada questionário, desenvolvemos estruturas de análise das variáveis escolhidas.

\section{A VARIÁVEL "NSE"}

A variável NSE serviu como entrada de dados, indicando a característica de cada aluno a partir de indicadores familiares de escolaridade, renda, situação de emprego e profissão. Tal variável foi definida como incontrolável, pois o aluno traz hábitos e costumes da família para o interior das escolas.

Decidimos trabalhar com esses dados por entender que é possível identificar uma relação desigual entre alunos vindos das classes sociais menos favorecidas e aqueles que, já na sua entrada no sistema de ensino, trazem maiores índices na sua bagagem cultural, ou seja, capital sociocultural - pressuposto que pode permitir maior eficiência no aprendizado em relação aos demais num mesmo grupo, numa mesma sala de aula. Há consenso na literatura de que o diagnóstico do desempenho inicial dos alunos deve ser considerado para futuras medidas de desempenho.

O questionário dos pais forneceu as informações necessárias para compor o NSE médio das escolas. Nesse questionário, o pai, mãe ou responsável respondeu aos itens referentes a: nível de escolaridade, acomodações internas da habitação familiar, número de aparelhos domésticos em geral, endereço residencial atual e principal ocupação (situação empregatícia).

Para obter o NSE, usamos os cálculos da tabela Abep/ Abipeme. Combinamos as informações obtidas nos questionários respondidos pelos pais com o Critério de Classificação Econômica Brasil. Essa opção de calcular o NSE médio das escolas pela Abep/Abipeme é um procedimento adotado pelo Laboratório de Estudos Descritivos (Loed-Unicamp).

Desse modo, foi gerada uma tabela de NSE e feita uma correlação de Pearson entre a medida de NSE cedida pelo Projeto Geres e a calculada pelo Critério Brasil, cujo resultado foi de $94 \%$, significando que a metodologia era adequada. 
Mais detalhes sobre a construção dessa tabela podem ser encontrados em Almeida (2009).

\section{A VARIÁVEL "TRABALHO DO PROFESSOR"}

O questionário do professor, aplicado a 416 professores, continha 124 itens. Como preza a literatura em Estatística, é sempre interessante, diante de uma considerável quantidade de dados, realizar uma análise exploratória dos mesmos.

\section{CONSTRUTOS: UMA ANÁLISE EXPLORATÓRIA DE DADOS}

Nessa análise, pudemos constatar uma divisão de temas nas questões em cinco partes: a primeira referia-se às políticas da escola, ao desenvolvimento do projeto político pedagógico e aos relacionamentos dos professores com a comunidade escolar de modo geral; a segunda, às influências do comportamento dos alunos e do ambiente escolar para melhora da aprendizagem; a terceira, às frequências de usos de materiais didáticos; a quarta parte, aos métodos e didática adotados; e a quinta, a características mais pessoais do professor.

Aqui vale um comentário: na pesquisa completa de Almeida (2009), essa análise exploratória foi mais ampla do que se poderia mostrar nos limites deste texto. O conceito de "construto", com respectivas bibliografias pertinentes à área (CARVALHO, 2003), foi adotado para a identificação dessas cinco partes. Além disso, utilizaram-se outras ferramentas estatísticas, como o teste Qui-Quadrado, para a análise da relação entre o tipo de escola (ciclada e não ciclada) e as respostas dos professores dentro daqueles construtos.

Verificou-se que as partes 2, 3 e 4 do questionário tiveram, estatisticamente, comportamento similar para escolas cicladas e as não cicladas, porém os percentuais dos grupos 1 e 5 apresentaram indicadores melhores para as cicladas. $\mathrm{O}$ quadro 1 , a seguir, ilustra alguns resultados desse grupo 5 , rotulado como um construto de "perfil do professor", envolvendo as questões 98 a 127: 
QUADRO 1 - Construto perfil do professor (G5)

\begin{tabular}{|c|c|c|}
\hline CONSTRUTO & ESPECIFICAÇÃO & $\begin{array}{l}\text { OPERACIONALIZACẼO COM } \\
\text { O ITEM DO QUESTIONÁRIO }\end{array}$ \\
\hline \multirow[t]{14}{*}{$\begin{array}{l}\text { Quinto grupo: } \\
\text { perfil do professor }\end{array}$} & $\begin{array}{l}\text { Trabalho de leitura que } \\
\text { desenvolve em sala de aula }\end{array}$ & Q98 \\
\hline & $\begin{array}{l}\text { Maneiras de alfabetizar/ } \\
\text { experiência de trabalho }\end{array}$ & Q99 \\
\hline & Escolaridade & Q100 \\
\hline & $\begin{array}{l}\text { Tipo de Instituição de } \\
\text { Ensino Superior }\end{array}$ & Q101 \\
\hline & Modalidade do curso de pós & Q102 \\
\hline & Carga horária de trabalho & Q106 \\
\hline & Anos de trabalho na escola & Q107 \\
\hline & Idade & Q110 \\
\hline & Renda familiar & Q114 \\
\hline & $\begin{array}{l}\text { Leitura de textos de } \\
\text { educação }\end{array}$ & Q116 \\
\hline & $\begin{array}{l}\text { Leitura de revista de } \\
\text { educação }\end{array}$ & Q118 \\
\hline & Frequência - cinema & Q119 \\
\hline & Ópera e concertos & Q121 \\
\hline & Livraria & Q124 \\
\hline
\end{tabular}

Os dados coletados no quinto grupo apontam uma tendência de índices mais elevados para as escolas cicladas. Os professores responderam a questões sobre gosto por leitura desde o tempo de aluno(a), trabalho com turma heterogênea, realização do curso de pedagogia, formação em instituições particulares ou públicas, pós-graduação, horas semanais de trabalho, idade, renda familiar, leituras atuais em educação, frequência a cinema (concertos, livrarias, etc.), dentre outros temas.

Não se pode, no entanto, perder de vista parte da questão central do estudo: esses dados do professor devem compor um quadro mais amplo com as outras variáveis, para se chegar às semelhanças e diferenças entre escolas cicladas e não cicladas em termos de desempenho. $\mathrm{E}$ a análise questão por questão pode não nos levar a uma caracterização mais objetiva dos professores. Assim, tratou-se de descartar as variáveis que não exerciam uma influência significativa no trabalho do professor e de agrupar as que se mostraram relevantes. Esse processo foi realizado por uma ferramenta estatística conhecida como Análise Fatorial.

\section{AGRUPAMENTO DE VARIÁVEIS POR ANÁLISE FATORIAL}

Em face de métodos de várias naturezas (ou técnicas) em pesquisas educacionais, julgamos de extrema importância 
aquelas que usam métodos quantitativos. No âmbito deste estudo, adotamos técnicas estatísticas agrupadas, que se convencionou chamar de Análise Multivariada.

Na definição de Hair et al. (2005), a análise multivariada refere-se ao uso de técnicas conjuntas de estatística avançada, que tratam as variáveis em pacotes, levando em conta muitas delas simultaneamente. Para Corrar, Paulo e Dias Filho (2007, p. 2), trata-se de "um conjunto de métodos estatísticos que torna possível a análise simultânea de medidas múltiplas para cada indivíduo, objeto ou fenômeno observado", ou seja, teorias que permitem um estudo de várias variáveis conjuntamente.

Antes de detalharmos uma das teorias em análise multivariada, é necessário voltarmos a uma ideia básica da Estatística, que é a correlação linear entre duas variáveis. Esse conceito determina o grau com que duas variáveis estão linearmente associadas, sendo que é uma medida que varia de -1 a 1 . Valores próximos desses extremos indicam correlação forte, enquanto valores próximos de 0 indicam fraca associação entre as variáveis ${ }^{1}$. No entanto, como indicam Aranha e Zambaldi (2008), tal medida não pode ser interpretada como um índice de "causalidade": correlação forte indica associação forte entre as variáveis, e não o fato de que uma variável necessariamente "cause" ou "determine a outra".

Entre as várias técnicas de análise multivariada (ver CORRAR, PAULO, DIAS FILHO, 2007, p. 91), optamos pela Análise Fatorial (AF), a mais adequada para situações com muitas variáveis, como é o caso da nossa pesquisa. Essa técnica "tem como um de seus principais objetivos tentar descrever um conjunto de variáveis originais através da criação de um número menor de dimensões ou fatores" (p. 74). As variáveis são agrupadas em função das suas altas correlações e tais agrupamentos tornam-se novas variáveis, em número menor e mais abrangente de fenômenos mais gerais. Essas novas variáveis se chamam fatores.

Cada fator é um grupo de variáveis que se relacionam entre si com maior intensidade. Por exemplo, se as variáveis A, B, C e D têm forte correlação entre si, elas compõem um fator X. O mesmo vale para variáveis $\mathrm{E}, \mathrm{F}$ e G, que compõem um fator $\mathrm{Y}$. Nesse exemplo, $\mathrm{X}$ e Y são as novas variáveis que provavelmente facilitarão as análises de fenômenos envolvendo as variáveis originais, e que alguns autores chamam de "variáveis latentes". 
2 Há ainda condições estatisticamente mais rigorosas, como teste de esfericidade de Bartlett, teste KMO - Kaiser-MeyerOlkin. Para mais detalhes, ver Almeida (2009)

3 O uso do software determina que as Soluções analíticas preditivas estão um passo à frente das ferramentas básicas de Banco Inteligente (BI) porque usam algoritmos e modelos estatísticos complexos, além de data mining e sistema de análise.
Trata-se de características mais gerais, não mensuráveis diretamente nos dados, mas implícitas nas variáveis A, B, C, D, E, F e $\mathrm{G}$ (as quatro primeiras explicitam o fator $\mathrm{X}$ e as três últimas, o fator Y).

Para que essa técnica seja bem utilizada (ARANHA, ZAMBALDI, 2008), o pesquisador deverá ter como hipótese mínima a quantidade de fatores comuns e apresentar uma expectativa teórica de qual fator deve influenciar as variáveis significativas na pesquisa em questão. Adaptamos de Corrar, Paulo e Dias Filho (2007) alguns dos principais objetivos do uso da AF: a) reduzir um grande número de variáveis a um número menor de fatores; b) selecionar alguma variável dentro de um grupo, o que, nesta pesquisa, foi feito com base nas maiores correlações, tendo sido descartadas as variáveis de menor interferência no processo; e c) criar condições de interpretabilidade dos relacionamentos entre as variáveis.

Há condições iniciais para se considerar se tal técnica é viável para o tratamento de dados. A primeira, como já foi visto, é a quantidade de variáveis e a possibilidade de interdependência delas entre grupos. Para confirmar ou não as hipóteses iniciais levantadas pelo pesquisador, utiliza-se a Análise Fatorial Confirmatória. Para os casos em que se conhece pouco ou nada sobre possíveis inter-relações entre as inúmeras variáveis envolvidas, pode-se recorrer à Análise Fatorial exploratória. Outra condição é a quantidade de dados. Hair et al. (2005) entendem que 100 ou mais elementos é um número normalmente satisfatório. Regra geral, é preciso ter pelo menos cinco vezes mais dados do que o número de variáveis analisadas ${ }^{2}$.

Visto que todas essas condições foram satisfeitas, ou se mostraram parcialmente adequadas, seguimos em frente com o uso da AF para análise do trabalho pedagógico dos professores. Procuramos não apenas agrupar variáveis mais associadas entre si, mas também identificar qual o grupo de variáveis originais que agregava a maior variância dos dados. Ou seja, identificar qual o fator (variável latente) que provavelmente interfere na eficiência da escola.

Nossas análises foram realizadas por meio do Statistical Package for the Social Sciences (SPSS) ${ }^{3}$. Dos dados coletados em 2005, foram selecionadas informações ou variáveis que, em conjunto, evidenciam o grau de comprometimento do professor com a 
atividade pedagógica. Admitindo que esse empenho possa interferir no aprendizado do aluno e, consequentemente, favorecer a maior ou menor proficiência média das escolas, tomou-se o fator que agrupa essas variáveis como uma das entradas de dados para as análises posteriores análises via Análise por Envoltória de Dados (DEA), da qual trataremos mais adiante.

O processo, para chegar aos fatores, passa por sucessivas análises recursivas, com cálculos e recálculos contínuos e a eventual exclusão de variáveis que não se correlacionam (correlação menor que 0,5 ). Outros procedimentos adotados foram: rotação varimax (que envolve correlações); matrizes anti-imagem e outras matrizes de correlação; e a "tabela de comunalidades", que corresponde "à proporção da variância de cada item observado, explicada pelo fator comum que o influencia (ou pelos fatores comuns, caso haja mais de um)" (ARANHA, ZAMBALDI, 2008, p. 48).

Nossas escolhas foram determinadas, portanto, pelos fatores e suas relações com as variáveis, a saber: participação/ desenvolvimento do projeto pedagógico da escola; escolaridade do professor; modalidade do curso de pós-graduação; número de anos em que o professor trabalha na escola; número de anos em que se dedica a essa ocupação; número de anos em que trabalha com a mesma série. Assim, os indicadores de desempenho focados no trabalho pedagógico do professor, calculado como "escore do professor", foram incluídos nas análises por escola.

A partir do objetivo da AF de reduzir o conjunto de variáveis originais, julgamos conveniente calcular escores fatoriais para cada sujeito pesquisado, como recomenda Malhotra (2006, p. 556), considerando que "um fator nada mais é que uma combinação linear das variáveis originais". Obtivemos, dessa forma, um resultado parcial com cada escore do professor e, em seguida, fizemos um recálculo para chegar ao escore médio de cada escola.

Ressaltamos a observação de Miranda (2006, p. 36) de que “o primeiro fator é o melhor resumo dos dados originais, já que é extraído de modo a preservar o máximo da variância inicialmente existente", pois consideramos que essa técnica se fortalece à medida que associamos o trabalho do professor às outras variáveis já utilizadas. 
No quadro 2, a seguir, observamos os fatores e os componentes numéricos de cada um dos grupos originais (G1, G2, G3, G4 e G5), podendo identificar as respostas que mais tiveram influência na composição do escore do professor. Os agrupamentos G2 e G3 foram eliminados pela AF.

\begin{tabular}{|c|c|c|c|}
\hline \multirow[t]{2}{*}{ FATORES } & \multicolumn{3}{|c|}{ COMPONENTES } \\
\hline & 1 & 2 & 3 \\
\hline $\begin{array}{l}\text { Como foi desenvolvido o projeto pedagógico } \\
\text { do professor desta escola neste ano (G1) }\end{array}$ & 0,064 & & \\
\hline Há quantos anos trabalha nesta escola (G5) & 0,468 & & \\
\hline $\begin{array}{l}\text { Há quantos anos trabalha nesta ocupação } \\
\text { (G5) }\end{array}$ & 0,422 & & \\
\hline $\begin{array}{l}\text { Há quantos anos o professor trabalha esta } \\
\text { série (G5) }\end{array}$ & 0,414 & & \\
\hline Nível de escolaridade do professor (G5) & & 0,539 & \\
\hline Modalidade da pós-graduação (G5) & & 0,497 & \\
\hline Com que frequência utiliza livro didático (G4) & & & 0,72 \\
\hline
\end{tabular}

Após a identificação dos fatores, foi necessário interpretar essa composição. Estes devem ser considerados fatores de representação com base nas cargas fatoriais. Assim, temos que o fator 1, ou fator principal, representou um indicador de avaliação do trabalho pedagógico do professor, comportando um conjunto de variáveis originais.

Essas variáveis agrupadas significaram a possibilidade de medir (numericamente) o trabalho pedagógico do professor pela AF. Esses dados foram obtidos cruzando-se informações sobre nível de escolaridade, tempo de trabalho na escola, carga horária, renda familiar e relacionamento com os colegas e com a direção. As variáveis que mais influenciam seu trabalho aparecem na primeira coluna da tabela anterior.

Pudemos observar que, após a elaboração do quadro de construtos, as decisões de seleção das variáveis que realmente identificavam o trabalho pedagógico do professor foram apontadas nos itens de número 1 a 124, com eliminação de alguns ${ }^{4}$; tais itens foram submetidos à $\mathrm{AF}$ e nortearam nossas análises.

Ainda de acordo com as respostas dos professores, destaca$10,14,15,16,19,40,42,54,57$, $58,74,79,80,97,98,99,100$, mos outras questões ${ }^{5}$, relacionadas ao tipo de escola (cicladas), que retratam e fortalecem a nossa hipótese de associação do 
trabalho do professor com o melhor desempenho (aprendizado) do aluno e, portanto, podem contribuir significativamente para maior eficiência da escola.

Esses fatores, calculados pela AF, podem servir como entradas de dados em outras técnicas operacionais, que neste caso foi a DEA. Vale realçar que os valores encontrados podem "representar um indicador de avaliação de determinado fenômeno que comporta um conjunto de variáveis originais" (REIS, 2009, p. 260).

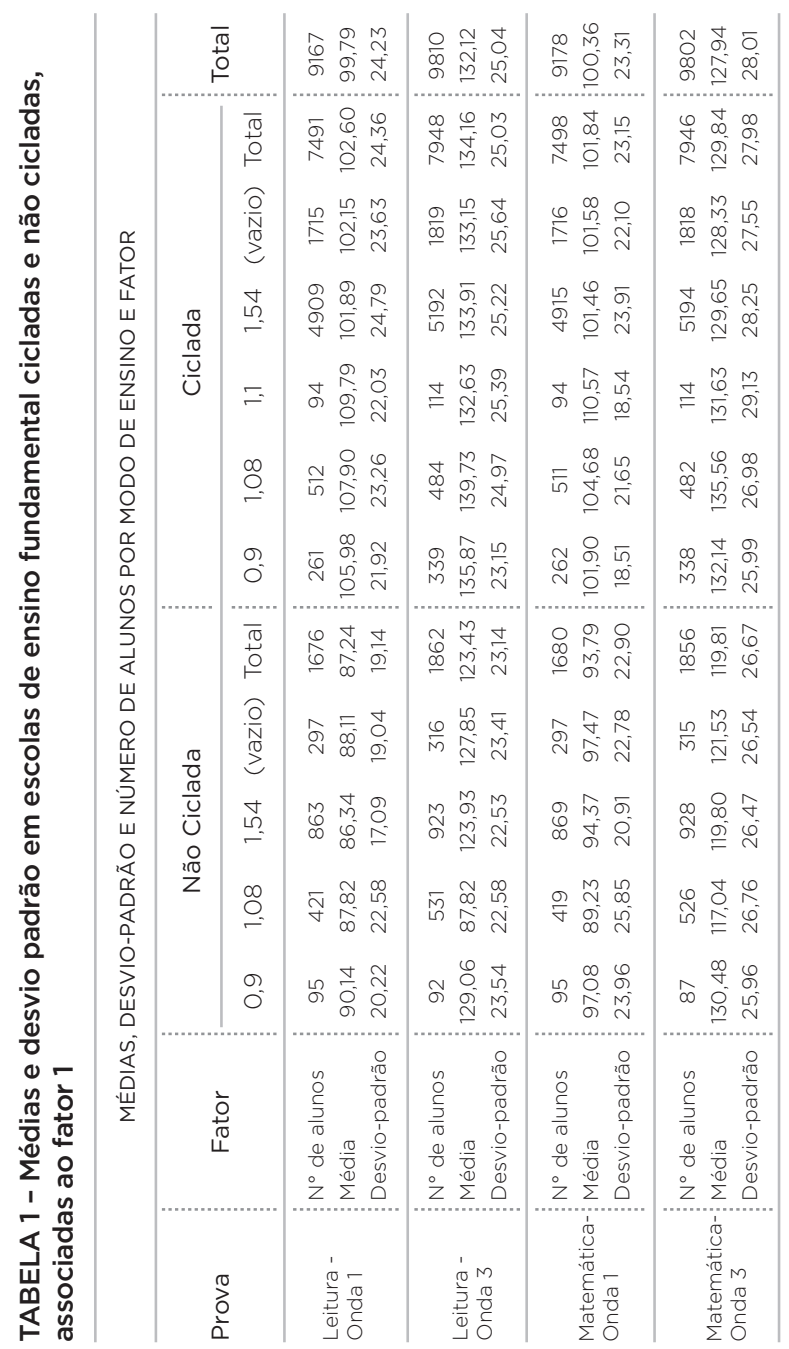


Observa-se, nesses índices calculados pela Análise Fatorial (AF), que o "fator" (valor numérico associado ao desempenho dos professores) é separado em quatro categorias: 0.9, 1.08, 1.1 e 1.54. Verifica-se também como influenciaram em maior escala as médias das proficiências das escolas cicladas comparativamente às não cicladas.

Com esse resultado numérico, extraímos os fatores que influenciaram e estão presentes nos recursos das escolas, e que serviram de entrada de dados para o cruzamento com as outras duas variáveis maiores do estudo, ou seja, NSE e proficiência das escolas. Falaremos desses valores mais adiante, quando cruzaremos as três variáveis maiores via DEA.

TESTES DE APLICAÇÃO:

\section{A PROFICIÊNCIA DOS ALUNOS}

Os testes de aplicação em leitura e matemática, realizados em 2005 e 2006, que mencionamos no início deste texto, não requerem um esforço de tratamento dos dados, como no caso do NSE e do trabalho do professor, já que as proficiências dos alunos foram objetivamente obtidas nas avaliações já mencionadas.

\section{A INTER-RELAÇÃO FINAL DAS VARIÁVEIS NSE, TRABALHO DO PROFESSOR E PROFICIÊNCIA MÉDIA DAS ESCOLAS: A FERRAMENTA DEA}

O uso crescente de técnicas operacionais envolvendo dados coletados em ambientes educacionais justifica-se pelas várias possibilidades de análise que oferecem, como também pela facilidade de uso e pela possibilidade de elaboração de cenários. O modelo de Análise por Envoltória de Dados (DEA) facilita a identificação de unidades eficientes e não eficientes e, desse modo, permite fazer comparações, neste caso, entre escolas cicladas e não cicladas.

Os momentos da presente pesquisa e as etapas descritas foram sistematicamente organizados para medir a eficiência do conjunto de escolas investigadas. A partir da aplicação da 
DEA, foi possível agrupar as escolas, com a vantagem de torná-las visíveis sem impor um padrão externo, mas considerando suas características locais. Dessa forma, as variáveis construídas pelo modelo foram sensíveis às condições de cada unidade escolar analisada.

A escolha da utilização de medidas de eficiência para escolas de ensino fundamental pública foi, em princípio, analisada com base em dimensões já estabelecidas para o ensino superior, no entanto alguns parâmetros utilizados podem, no decorrer das análises, ser coincidentes e apontar os mesmos caminhos para unidades escolares, sejam elas de ensino fundamental ou superior.

\section{CONSIDERAÇÕES FINAIS}

Os resultados obtidos pela análise de eficiência produtiva pela DEA mostram que esse método pode ser de grande utilidade para efetuar avaliações dentro da organização escolar de ensino fundamental. Neste trabalho, a partir dos resultados, procuramos despertar a atenção dos estudiosos da área educacional para essa ferramenta operacional de análise de eficiência produtiva em problemas multidimensionais que, até o momento, ainda é pouco explorada.

Outro fator importante é a escolha do modelo a ser utilizado para análise, que deve ser adequado aos objetivos que se pretenda atingir. Caso contrário, pode se obter um grupo de unidades eficientes que não represente padrões de referência para efetuar possíveis inferências ou comparações (COOK et al.,1992).

Dessa análise contextual, consideramos que nossa pesquisa se alinha a outras, como a de Rodrigues (2005), Poli (2007), Belloni (2003), Miranda (2006) e Nunes (2002), que tiveram como preocupação a utilização e consolidação de uma metodologia que utiliza meios da pesquisa operacional para, dentre outros resultados, fomentar o debate acerca da utilização de recursos tecnológicos no contexto das avaliações de desempenho em organizações escolares do ensino fundamental.

Na tentativa de contribuir para a difusão dessa metodologia, apresentam-se os procedimentos adotados neste estudo: 
1. buscou-se atender aos princípios e características de uma avaliação quantitativa longitudinal;

2. estabeleceram-se medidas de desempenho em Leitura e Matemática de escolas públicas cicladas e não cicladas;

3. evidenciou-se a importância dos insumos (recursos) oferecidos pelo trabalho pedagógico dos professores para o desempenho do aluno, tendo como referências as avaliações em Leitura e Matemática;

4. verificou-se a eficiência da escola com base nos resultados em termos de produtividade, objetivos atingidos, projeto pedagógico construído e aplicado mediante a definição de metas e o próprio planejamento escolar, de acordo com o seu tipo de escola;

5. associou-se o trabalho do professor (recurso da escola) ao desempenho das escolas, apontando resultados da eficiência de cada uma delas.

Em linhas gerais, nossos indicadores de eficiência produtiva das escolas públicas, cicladas e não cicladas, possibilitaram, em nossa pesquisa: (a) identificar as escolas eficientes inserindo recursos e obtendo resultados; (b) identificar associações fortes e fracas entre as variáveis, o trabalho do professor associado à proficiência média da escola em Leitura e Matemática em cada escola investigada, consideradas como significativas para esse estudo; (c) identificar possíveis distanciamentos entre as mais e menos eficientes DMUs (escolas cicladas e não cicladas).

O uso da Análise por Envoltória de Dados permitiu construir indicadores de desempenho em Leitura e Matemática (resultados), condição que assegura a aferição de produtividade em ambiente escolar público, a partir da utilização de várias coletas de dados aplicadas em momentos diferentes nas mesmas escolas e para os mesmos alunos.

Essa construção longitudinal empregada em nossa pesquisa foi operacionalmente consistente e serviu para evitar a desvantagem apontada na literatura com relação à modelagem DEA, ou seja, que seus resultados estáticos e sua aplicação, em um único período, não captam as interações entre as políticas de curto prazo e de longo prazo e o desempenho discente.

Verificamos, nessa base de dados, que existem diferenças entre as escolas cicladas e não cicladas, mas advertimos que 
esses resultados não devem ser extrapolados além do conjunto das escolas pesquisadas, já que a modelagem DEA não preconiza a generalidade.

Por último, e não menos importante, vale lembrar que a opção por métodos em pesquisa qualitativa pode ser articulada à metodologia deste estudo. A título de exemplo ou indicação para pesquisa futura, algumas escolas seriam selecionadas, aleatoriamente ou por meio da classificação de seus níveis de eficiência, e teriam suas características exploradas por uma ou mais ferramentas/recursos da pesquisa qualitativa.

\section{REFERÊNCIAS BIBLIOGRÁFICAS}

ALMEIDA, Ivanete Bellucci. Análise do desempenho de escolas públicas cicladas e não cicladas pertencentes ao ensino fundamental. Tese (Doutorado) - Faculdade de Educação - Unicamp, Campinas, 2009.

ARANHA, F.; ZAMBALDI, F. Análise fatorial em administração. São Paulo: Cengage Learning, 2008.

BARBETTA, P. A. Estatística aplicada às ciências sociais. Florianópolis: UFSC, 2003.

BELLONI, José Ângelo. Uma metodologia de avaliação da eficiência produtiva de universidades federais brasileiras. Tese (Doutorado em Engenharia de Produção) - Universidade Federal de Santa Catarina, Florianópolis, 2003.

BERTAGNA, R. H. Progressão Continuada: limites e possibilidades. Tese (Doutorado em Educação) - Universidade Estadual de Campinas, Campinas, 2001.

BEZERRA. F. A. Análise Fatorial. In: CORRAR, L. J.; PAULO, E.; DIAS FILHO, J. M. F. (Coord.). Análise multivariada: para cursos de administração, ciências contábeis e economia. São Paulo: Atlas, 2007. p. 73-130. cap. 2

BOLETIM GERES. Belo Horizonte: UFMG, ano 1, n. 1, 2005.

COOK, W. D. et al. Prioritization models for frontier decision making units in DEA. European Journal of Operational Research, v. 59, n. 2, p. 319-23, 1992.

CORRAR, L. J.; PAULO, E; DIAS FILHO, J. M. (Coord.). Análise multivariada: para cursos de administração, ciências contábeis e economia. São Paulo: Atlas, 2007.

HAIR, J. F.; ANDERSON, R. E.; TATHAM, R. L.; BLACK, W. C. Análise multivariada de dados. 5. ed. Porto Alegre: Bookman, 2005.

Multivariate data analysis. 5. ed. New Jersey: Prentice Hall, 1998.

LEE, V.; SMITH, J. Social support and achievement for young adolescents in Chicago: The role of school academic press. American Educational Research Journal, v. 36, n. 4, p. 907-45, 1999. 
MALHOTRA, N. Pesquisa de marketing: uma orientação aplicada. 4. ed. Tradução de Laura Bocco. Porto Alegre: Bookman, 2006.

MIRANDA, E. C. M. O Saeb-2003 no Estado de São Paulo: um estudo multinível (HLM). 2006. Dissertação (Mestrado em Educação) - Universidade Estadual de Campinas, Campinas, 2006.

NUNES, N. Avaliação da eficiência produtiva de organizações educacionais.

Florianópolis: Insular, 2002.

POLI, E. C. Estudo longitudinal em Matemática: possibilidades e leitura de uma realidade do Ensino Fundamental. 2007. Tese (Doutorado em Educação) Universidade Estadual de Campinas, Campinas, 2007.

REIS, E. Análise fatorial. In: FAVERO, L. P.; BELFIORE, P.; SILVA, F. L.; CHAN, B. L. (Org.). Análise de dados: modelagem multivariada para tomada de decisões. Rio de Janeiro: Elsevier, 2009. cap. 7.

RODRIGUES, S. C. Construção de uma metodologia alternativa para avaliação das escolas públicas de ensino fundamental através do uso da Análise por Envoltória de Dados (DEA). 2005. Tese (Doutorado) - Universidade Estadual de Campinas, 2005.

TRIOLA, M. F. Introdução à estatística. Rio de Janeiro: LTC, 2005.

\author{
IVANETE BELLUCCI PIRES DE ALMEIDA \\ Fatec Indaiatuba \\ ivanete@fatecindaiatuba.edu.br
}

FRANCISCO CARLOS BENEDETTI

Fatec Indaiatuba

fcbenedetti@terra.com.br 